\title{
ACTIVE CONTROL AND ADAPTIVE OPTICS FOR OPTICAL INTERFEROMETERS
}

\author{
FRITZ MERKLE \\ European Southern Observatory \\ Karl-Schwarzschold-Str. 2, D-8046 Garching \\ Federal Republic of Germany
}

Long baseline interferometry requires the full phasing of a telescope array. Especially for future arrays with large unit telescopes active control systems are mandatory. Adaptive optics can be applied for real-time phase compensation of the individual pupils due to atmospheric distortions. Additional to phasing of the individual pupils of independently mounted telescopes, the whole array has to be phased, including pupil position corrections due to pupil foreshortening and shift effects in order to reach a reasonable phased field-ofview.

From the beginning it can be excluded that a linear array and its large unit telescopes offer passive stability sufficient for coherent beam combination in interferometry. The finally tolerable vibrational amplitudes are less than a tenth of a wavelength, which is not achievable, even in the 10 to $20 \mu \mathrm{m}$ wavelength ranges. Therefore, it is important to develop techniques for active stabilization of the light beams, after they have left the unit telescopes. Nevertheless, the unit telescopes should be as stable as possible, in order to limit these stabilization efforts. For pathlength control of the telescope and combining optics, laser interferometers with closed loop active stabilization of the opto-mechanical system are proposed.

In order to guarantee phasing of the desired field-of-view, off-axis as well as on-axis for an array of telescopes, the Lagrange invariants of individual telescopes including their combining trains must be equal, and additionally the overall Lagrange invariant of the array must be conserved. The co-phasing of an array of independent telescopes differs significantly from the requirement for telescopes arranged in the same mechanical mount (like multimirror telescope type interferometers). This has a major impact on the optical design of the final combination optics. In order to maintain the necessary geometrical scaling of the lateral pupil geometry, the separation of the pupil images at the combining optics has to be adjusted according to the zenith angle. Additionally, the longitudinal pupil position needs a correction due to the shift of the relative locations of the optical elements in the combining train with respect to the wavefronts. Besides these pupil corrections, which determine the off-axis phasing, the overall on-axis phase difference between the telescopes to be combined has to be compensated. In order to perform the necessary pathlength compensation an optical delay line is proposed for each telescope. These pathlength and pupil corrections are mandatory for interferometers composed of large telecopes.

Obtaining diffraction limited images or spectra from the ground, either with a single large pupil or with two or more smaller pupils, suffers basically from the same limitation: the fluctuations of the index of refraction of the atmosphere due to turbulence. For long baseline 
interferometry the gain of large apertures is only given in combination with adaptive optics. Otherwise the signal-to-noise ratio will not be improved compared with interferometers with smaller apertures. Phasing the individual pupils, at least partially, is a fundamental requirement for the efficient use of a large aperture interferometer. Large scale atmospheric wavefront aberrations have to be corrected by a measurement of the relative phase between the telescopes to be combined. The same correction system which is used to compensate phase fluctuations due to mechanical vibrations could be applied. Fringe tracking will allow long integration times resulting in a considerable sensitivity gain. 